\section{El consentimiento informado $y$ la vigilancia nutricional en los escolares peruanos}

\section{Tarqui-Mamani, Carolina}

Instituto Nacional de Salud, Departamento de Medicina Preventiva y Salud Pública de la Universidad Nacional Mayorde San Marcos.E-mail carobtm@gmail.com

\section{Alvarez-Dongo, Doris \\ Instituto Nacional de Salud}

\section{Espinoza-Oriundo, Paula}

Instituto Nacional de Salud

\section{PALAVRAS-CHAVE:}

Alimentación Escolar;

Consentimiento Informado, Servicios de Salud Escolar

Introducción. La vigilancia ${ }^{1}$ nutricional constituye la piedra angular para disponer de información oportuna que permita mejorar la situación nutricional de los escolares ${ }^{2}$. Objetivo: Identificar los aspectos vinculados al consentimiento informado -CI- aplicado en la vigilancia nutricional de los escolares peruanos. Método: Se realizó un muestreo probabilístico, multietápico y estratificado. La muestra incluyó 8628 estudiantes de primaria distribuidos en 719 instituciones educativas -IE- (públicos y privados). Se realizó la revisión de las pautas éticas y aplicación del consentimiento informado. Se realizó la evaluación de la lonchera escolar y la medición de la concentración de yodo en orina y en sal ${ }^{3}$. Se solicitó la autorización del Ministerio de Educación, Dirección Regional de Salud, directores de IE del director de la institución educativa, y en el caso que acepte que se realice la evaluación de los escolares, se procedió a solicitar el asentimiento informado por escrito de los escolares de 9 años a más y el respectivo consentimiento informado de los padres del escolar. Al finalizar la aplicación del consentimiento informado se entregó una copia del formato a cada uno de los padres. Resultados: Se realizó la evaluación de 8185 escolares, se observó que EL 100\% de los cuestionarios contienen el formato de CI firmado y con la huella dactilar de los padres o apoderados. CI incluyó, los objetivos, procedimientos, uso de la información, confidencialidad y datos de referencia telefónica del INS. La identificación de los escolares fue custodiado por el equipo técnico y se empleó un código identificador para el análisis de laboratorio. Se encontró que la prevalencia del uso de loncheras fue 58,7 \% (IC 95\%:4,6;6,3). La prevalencia de loncheras saludables fue 5,4\% (IC95\%: 4,6;6,3). Del total de niños, 5,6 \% tuvieron loncheras saludables y 5,2 \% del total de niñas tuvieron loncheras saludables. El 6,3\% de los niños que residieron en la zona urbana tuvieron lonchera saludable y $0,8 \%$ en la zona rural. Los niños que residieron en Lima Metropolitana $(8,1 \%)$ y la selva $(8,1 \%)$ tuvieron loncheras saludables. A nivel nacional, la mediana de yoduria en los escolares fue 258,53 ug/L, siendo mayor en los varones $(265,90 \mathrm{ug} / \mathrm{L})$ que en las mujeres $(250,77 \mathrm{ug} / \mathrm{L})$. La mediana de yoduria en el área urbana fue mayor $(289,89 \mathrm{ug} / \mathrm{L})$ que en el área rural $(199,67 \mathrm{ug} / \mathrm{L})$. Mientras que en los colegios privados fue $315,48 \mathrm{ug} / \mathrm{L}$ y en los públicos fue $241,56 \mathrm{ug} / \mathrm{L}$ ( $<<0,001)$ La mediana de yodo en sal fue $28,69 \mathrm{mg} / \mathrm{kg}$. El $23,1 \%$ de las muestras de sal, tuvieron $<15 \mathrm{mg} / \mathrm{Kg}$ de yodo.

\section{REFERÊNCIAS:}

[1] OMS. Pautas de la OMS sobre la ética en la vigilancia de la salud pública. Washington, D.C.: Organización Panamericana de la Salud; 2017

[2] Congreso de la República Ley de promoción de la alimentación saludable para niños, niñas y adolescentes [Ley peruana $\mathrm{N}^{\circ}$ 30021]. May 17 2013. Lima: MINSA; 2013 [Citado 27 Feb 2016]. Disponible en: <http://www.minedu.gob.pe/files/6231_201305171019.pdf.>

[3] World Health Organization (WHO), United Nations Children's Fund (UNICEF), International Council for Control of Iodine Deficiency Disorders (ICCIDD). Assessment of iodine deficiency disorders and monitoring their elimination A guide for programme managers. 3rd ed. WHO/UNICEF/ICCIDD. Geneva:WHO, 2008 